\title{
Application of a Strong Tracking Finite-Difference Extended Kalman Filter to Eye Tracking
}

\author{
Zutao Zhang and Jiashu Zhang \\ Southwest Jiaotong University
}

China

\section{Introduction}

Since eye tracking was first introduced by Mowrer in 1936, it has been gaining in popularity over the past decades as a window into observers' visual and cognitive process. For instance, researchers have utilized eye tracking to study behavior in such domains as driver fatigue detection (Qiang et al., 2004; Horng et al., 2004; Dong et al., 2004 ), eye typing for helping users with movement disabilities interact with computers (Majaranta \& Raiha, 2002), eye tracking analysis of user behavior in WWW search (Laura et al., 2004), using eye tracking techniques to study collaboration on physical tasks for medical research, VR system for measuring inspection methods, and image scanning (Noton \& Stark, 1971). Above all applications, two types of human-computer interfaces utilize eye tracking, passive and active interfaces. Passive interfaces monitor the user's eye movements and automatically adapt themselves to the user. For example in driver fatigue detection, the researchers track the driver eyes to fatigue detection, because the human eyes express the most direct reaction when dozing, inattention and yawning. On the other hand, Active interfaces allow users to explicitly control the interface though the use of eye movements. For example, eye typing has users look at keys on a virtual keyboard to type instead of manually depressing keys as on a traditional keyboard (Majaranta \& Raiha, 2002; Takehiko et al., 2003). Such active interfaces have been quite effective at helping users with movement disabilities interact with computers. Not surprisingly, eye tracking has attracted the interest of many researchers, and eye trackers have been commercially available for many years (Qiang et al., 2004; Horng et al., 2004; Takehiko et al., 2003; John et al., 2005).

In the past decades, many researchers have paid attention to the eye tracking in human computer interaction. There have been many methods that support non-invasive eye tracking. In (Li et al., 2005), all of these eye tracking algorithms can be classified into two approaches: feature-based and model-based approaches. Feature-based approaches detect and localize image features related to the position of the eye. A commonality among featurebased approaches is that a criteria (e.g., a threshold) is needed to decide when a feature is present or absent. The determination of an appropriate threshold is typically left as a free parameter that is adjusted by the user. The tracked features vary widely across algorithms but most often rely on intensity levels or intensity gradients. For example, in infrared (IR) images created with the dark-pupil technique, an appropriately set intensity threshold can 
be used to extract the region corresponding to the pupil. The pupil center can be taken as the geometric center of this identified region. The intensity gradient can be used to detect the limbus in visible spectrum images (Zhu \& Yang, 2002) or the pupil contour in infrared spectrum images (Tohno et al., 2002). In (Qiang et al., 2004; Zhu et al., 2002; Ebisawa, 1995; Morimoto \& Flickner, 2000), several active IR based eye trackers were proposed. The authors thought that eye tracking based on the active remote IR illuminations is a simple and effective approach. But most of them require distinctive bright pupil effect to work well because they all track the eyes by tracking the bright pupils. Qiang et al. has also made significant improvement of eye tracking over existing techniques (Qiang et al., 2004; Zhu et al., 2002). However, their methods need IR eye detector, or bright pupils and steady illumination. Their eye tracking method using Kalman filtering is linear system estimation algorithm. In realistic driving environments, the eye motion is the high nonlinearity of the likelihood model, the stand Kanlman filter is no longer optimal.

On the other hand, model-based approaches do not explicitly detect features but rather find the best fitting model that is consistent with the image. For example, integrodifferential operators can be used to find the best-fitting circle (Daugman, 1993) or ellipse (Nishino \& Nayar, 2004) for the limbus and pupil contour. Michael Chau and Margrit Betke use correlation with an online template to eye tracking in (Michael \& Margrit, 2005). The authors (Horng et al., 2004) use the dynamic templates for eye tracking. After finding the eye templates, they are used for eye tracking by template matching. And the minimum value within the search area is the most matching position of the eye. The model- based approach can provide a more precise estimate of the pupil center than a feature-based approach given that a feature-defining criteria is not applied to the image data.

Eye tracking has not reached its full potential even though the general-purpose eye tracking technology has been explored for decades. The first obstacle to integrating these techniques into human-computer interfaces is that they have been too expensive for routine use. Currently, a number of eye trackers are available on the market and their prices range from approximately 5,000 to 40,000 US Dollars (Li et al., 2005). The second factor is that it's very difficult to model to eye tracking because of the eye motion being the high nonlinearity. The third factor is the robustness of eye tracking should be improved because of the variety of head and eyes moving fast, external illuminations interference and realistic lighting conditions. The accuracy of eye tracking can't satisfy the realistic requirement of HCI.

To tackle some of those problems, we propose a strong tracking finite-difference extended Kalman filter algorithm to eye tracking. In this paper, a strong tracking factor is introduced to modify prior covariance matrix to improve the accuracy of the algorithm. And the finitedifference method is presented to calculate partial derivatives of nonlinear functions to eye tracking. At the same time, we overcome the eye tracking modeling in nonlinear system. The last experimental results show that the average correct rate of eye tracking can achieve $99.4 \%$ on three video.

The organization of the paper is as follows. Strong Tracking Finite-Difference Extended Kalman Filter algorithm is given in next section. Section 3 gives STFDEKF based Eye Tracking algorithm and experimental results. Final conclusion is in section 4.

\section{Strong tracking finite-difference extended Kalman filter}

Extended Kalman filter (EKF) is one of the most common and popular filtering approach in nonlinear target tracking and state estimation. It includes state estimation of a nonlinear 
dynamic system, parameters estimation for nonlinear system identification and dual estimation where both states and parameters are estimated simultaneously. However, EKF simply linearizes all nonlinear functions to the first order by using the Talyor series expansions. At the same time, EKF may cause more errors for the nonlinear system while estimating system state and its variance. Moreover, the linearization may lead to divergence of filtering process. In a nonlinear mismatched model and limited applications scope, EKF filter will lead the divergence problem of state estimation. For these reasons, two improved EKF algorithms are introduced to tackle some of those problems.

\subsection{Suboptimal fading extended Kalman filter}

In this section, an adaptive extended Kalman filter - a suboptimal fading extended Kalman filter (SFEKF) is presented. The derivation of the filter is presented (Zhou et al., 1991; Zhou et al., 1990) in detail. SFEKF has the following good properties: 1) lower sensitivity to the statistics of the initial states and the statistics of the system and/or measurement noise, 2) stronger tracking ability to the suddenly changing states and bias no matter whether the filter operates in dynamic or stationary fashion, 3) acceptable computational complexity.

Considering a class of nonlinear discrete-time dynamical system,

$$
\begin{gathered}
x_{k+1}=f\left(x_{k}, u_{k}, v_{k}\right) \\
y_{k}=g\left(x_{k}, w_{k}\right)
\end{gathered}
$$

where, $x_{k}$ is the state vector, $y_{k}$ is the measurement vector, $u_{k}$ is control input vector, $v_{k}$ is process noise and $w_{k}$ is measurement noise. $v_{k}$ and $w_{k}$ are statistically independent. The equations of mean and covariance are as follow.

$$
\begin{gathered}
E\left[v_{k}\right]=q_{k}, \quad \operatorname{cov}\left[v_{k}, v_{j}\right]=Q_{k} \delta(k-j) \\
E\left[w_{k}\right]=r_{k}, \quad \operatorname{cov}\left[w_{k}, w_{j}\right]=R_{k} \delta(k-j)
\end{gathered}
$$

The extended Kalman filter is based on the assumption that sensor noises and, propagation errors are driven by zero-mean, Gaussian-distributed, white, random process. Retaining only the first-order terms in the Taylor series expansion, one obtains

$$
\left\{\begin{array}{l}
x_{k+1} \approx f\left(\hat{x}_{k}, u_{k}, q_{k}\right)+F_{x}(k)\left(x_{k}-\hat{x}_{k}\right)+F_{v}(k)\left(v_{k}-q_{k}\right), \\
y_{k} \approx g\left(\bar{x}_{k}, r_{k}\right)+G_{x}(k)\left(x_{k}-\hat{x}_{k}\right)+G_{w}(k)\left(w_{k}-r_{k}\right),
\end{array}\right.
$$

where $F_{x}(k)$ and $F_{v}(k)$ are the partial derivatives of $f(\bullet)$ to $x$ and $v, G_{x}(k)$ and $F_{w}(k)$ are the partial derivatives of $f(\bullet)$ to $x$ and $w$.

So the suboptimal fading extended Kalman filter (SFEKF) is deduced as follows:

The predicted state estimation equations are

$$
\begin{gathered}
\bar{x}_{k+1}=f\left(\hat{x}_{k}, u_{k}, v_{k}\right) \\
\bar{y}_{k}=g\left(\bar{x}_{k}, r_{k}\right)
\end{gathered}
$$


The predicted covariance estimation equations are

$$
\bar{P}_{k+1}=\lambda(k+1) F_{x}(k) \hat{P}_{k} F_{x}(k)^{T}+F_{v}(k) Q_{k} F_{v}(k)^{T}
$$

Where $\lambda(k+1) \geq 1$ is the suboptimal fading factor, which is used to fade the bypast datum and adjust predictable state estimation covariance matrix.

With this model (Zhou et al., 1993), $\lambda(k+1)$ can be directly determined as follows:

Where

$$
\lambda(k+1)=\left\{\begin{array}{l}
\lambda_{0}, \lambda_{0} \geq 1 ; \\
1, \lambda_{0}<1
\end{array}\right.
$$

$$
\begin{gathered}
\lambda_{0}=\operatorname{tr}[N(k+1)] / \operatorname{tr}[M(k+1)], \\
N(k+1)=V_{0}(k+1)-G_{x}(k) F_{v}(k) \bullet F_{v}(k)^{T}-G_{w}(k) R_{k} G_{w}(k)^{T}, \\
M(k+1)=G_{x} F_{x}(k) \hat{P}_{k} F_{x}^{T}(k) G_{x}^{T}(k) . \\
V_{0}(k+1)=\frac{1}{k} \sum_{j=1}^{k} \gamma_{j} \gamma_{j}^{T}=\left\{\begin{array}{l}
G_{x}(0) \hat{P}_{0} G_{x}(0)+G_{w}(0)^{T}, k=0 ; \\
\frac{\rho V_{0}(k)+\gamma_{j} \gamma_{j}^{T}}{1+\rho}, k \geq 1
\end{array}\right.
\end{gathered}
$$

with $0 \leq \rho \leq 1$ is the preselected forgetting, it may be selected according to the real processes. For fast changing processes, a smaller $\rho$ should be selected, and vice versa. As that pointed out in the paper (Zhou et al., 1993), $\lambda(k+1)$ is insensitive to the value of $\rho$.

\subsection{Strong tracking finite-difference extended Kalman filter}

Deriving the ideas in papers (Fan et al., 2006; Zhou et al., 1997), the authors proposed a finite-difference method to replace partial derivatives of nonlinear functions. From further improving the self-covariance and between-covariance, we obtain the algorithm based on strong tracking filter-difference enhanced kalman filter.

We adopt cholesky to decompose $Q_{k}, R_{k}, \bar{P}_{K}, \hat{P}_{k}$,

$$
\begin{aligned}
& Q_{k}=S_{v} S_{v}^{T}, R_{k}=S_{w} S_{W}^{T}, \\
& \bar{P}_{k}=\bar{S}_{x} \bar{S}_{x}^{T}, P_{k}=\hat{S}_{x} \hat{S}_{x}^{T} .
\end{aligned}
$$

central difference of partial derivative in nonlinear function $F x(k)$ :

$$
F_{x}(k)=\left\{f_{i j}\right\}=\left\{\left(f_{i}\left(x_{k, j}+\Delta x_{k, j}, u_{k}, q_{k}-f\left(x_{k, j}-\Delta x_{k, j}, u_{k}, q_{k}\right)\right) / 2 \Delta x_{k, j}\right)\right\}
$$


Where $\Delta \hat{x}_{k, j}=h \hat{s}_{x, j}, h$ is the step adjustment coefficient; $\hat{S}_{x, j}$ represents the $\mathrm{j}$ column of $\hat{S}_{x}$, then

$$
\begin{gathered}
F_{x}(k) \hat{S}_{x}=S_{x \bar{x}}=\left\{\left(f\left(\hat{x}_{k}+h s_{x, j}, u_{k}, q_{k},\right)-f_{i}\left(\hat{x}_{k}+h s_{x}, j, u k, q_{k}\right)\right) / 2 h\right\} . \\
F_{v}(k) S_{v}=S_{x v}=\left\{\left(f_{i}\left(\hat{x}_{k}, u_{k}, q_{k},+h s_{v, j}\right)-f_{i}\left(\hat{x}_{k}, u_{k}, q_{k}-h s_{v, j}\right)\right) / 2 h\right\}, \\
G_{x}(k) \bar{S}_{x}=S_{y \bar{x}}=\left\{\left(g_{i}\left(\bar{x}_{k}+h \bar{S}_{x}, j, r k\right)-g_{i}\left(\bar{x}_{k}-h \bar{S}_{x}, j, r k\right)\right) / 2 h\right\}, \\
G_{w}(k) S_{w}=S_{y w}=\left\{\left(g_{i}\left(\bar{x}_{k, r k}+h s_{w, j}-g_{i}\left(\bar{x}_{k, r k}-h s_{w, j}\right)\right) / 2 h\right)\right\}
\end{gathered}
$$

The predicted covariance matrix, gain matrix and covariance estimate of suboptimal fading extended Kalman filter (SFEKF) are mended as follows

$$
\begin{aligned}
& \bar{P}_{k+1}=\lambda(k+1) F_{x}(k) \hat{P}_{k} F_{x}(k)^{T}+F_{v}(k) Q_{k} F_{v}(k)^{T}= \\
& \lambda(k+1) F_{x}(k) \hat{S}_{x} \hat{S}_{x}^{T} F_{x}(k)^{T}+F_{v}(k) \hat{S}_{v} \hat{S}_{v}^{T} F_{v}(k)^{T}= \\
& \lambda(k+1) S_{x \hat{x}} S_{x \hat{x}}^{T}+S_{x v} S_{x v}^{T} ; \\
& K_{k+1}= \bar{P}_{k} G_{x}(k)^{T}\left[G_{x}(k) \bar{P}_{k} G_{x}(k)^{T}+G_{w}(k) R_{k} G_{w}^{T}(k)\right]^{-1}= \\
& \bar{S}_{x} \bar{S}_{x}^{T}\left(S_{y \bar{x}} S_{x}^{-1}\right)^{T}\left[S_{y \bar{x}} S_{y \bar{x}}^{T}+S_{y w} S_{y w}^{-T}\right]^{-1}=\bar{S}_{x} S_{y \bar{x}}{ }^{T}\left[S_{y \bar{x}} S_{y \bar{x}}{ }^{T}+S_{y \bar{w}} S_{y w}{ }^{-T}\right]^{-1} \\
& \hat{P}_{k+1}= \\
& {\left[I-K_{k+1} G_{x}(k) \bar{P}_{k+1}\right]=} \\
& \bar{S}_{x} \bar{S}_{x}^{T}-K_{k+1} G_{x}(k) \bar{S}_{x} \bar{S}_{x}^{T}= \\
& \bar{S}_{x} \bar{S}_{x}^{T}-\bar{S}_{x} \bar{S}_{y \bar{x}}^{T} K_{k+1}^{T}-K_{k+1} \bar{S}_{y \bar{x}}^{T} \bar{S}_{x}^{T}+\bar{S}_{x} \bar{S}_{y \bar{x}}^{T} K_{k+1}^{T}= \\
& \bar{S}_{x} \bar{S}_{x}^{T}-\bar{S}_{x} \bar{S}_{y \bar{x}}^{T}-K_{k+1} \bar{S}_{y \bar{x}}^{T} \bar{S}_{x}^{T}+ \\
& K_{k+1} \bar{S}_{y \bar{x}} \bar{S}_{y \bar{x}}^{T} K_{k+1}^{T}+K_{k+1} S_{y w w} S_{y w}^{T} K_{k+1}^{T}= \\
& {\left[\bar{S}_{x}-K_{k+1} \bar{S}_{y \bar{x}} K_{k+1} S_{y w}\right] \times\left[\bar{S}_{x}-K_{k+1} \bar{S}_{y \bar{x}} K_{k+1} S_{y w}\right]^{T} }
\end{aligned}
$$

From before-mentioned deduction, we can infer to that all calculations of above include the process noise impact and the error problem of model linearization. The step number which nonlinear function is linearized also changes with last time covariance matrix, process noise and observation noise. The filter becomes very simple because of replacing partial derivatives calculation using finite-difference value. The new strong finite-difference Kalman filter (STFDEKF) has more accuracy and covariance estimation, and improves the 
robustness of target tracking. The last experiment results show that the STFDEKF can be used for the high nonlinear stochastic systems such as eye tracking.

\section{STFDEKF based eye tracking and results}

In this section, we develop the following eye tracking using STFDEKF. Because the eye motion is the high nonlinearity of the likelihood model, it's very difficult to model human eye movement dynamics. In our tracking system, the following nonlinear equations are used to model the eye movement dynamics.

$$
\begin{gathered}
x=x_{0}+v t+\frac{1}{2} a t^{2} \\
\dot{x}_{k+1}=v_{0}+A_{k} \sin \left(\omega_{k} t\right) \\
a_{k+1}=\ddot{x}_{k+1}=A_{k} \omega_{k} \cos \left(\omega_{k} t\right)
\end{gathered}
$$

where, the initial value $x_{0}$ and $v_{0}$ are zero. The acceleration $a$ follows the sine distribution, and $a$ will be considered process noise $\left(v_{k}\right)$, respectively $A_{k}=0.08 \mathrm{~m} / \mathrm{s}$ and $\omega_{k}=\pi \mathrm{rad} / \mathrm{s}$. The proposed eye tracking experiment is developed in platform of OPEN CV. Our system uses a ViewQuest VQ680 video camera to capture human images. The experiment is tested on a Pentium III 1.7G CPU with 128MB RAM. Eye tracking based on the proposed method can reach 10 frames per second. The format of input video is $352 \times 288$. Fig. 1 represents the eye tracking using STFDEKF algorithm. The Correct Rate of eye tracking is shown in Table.1.

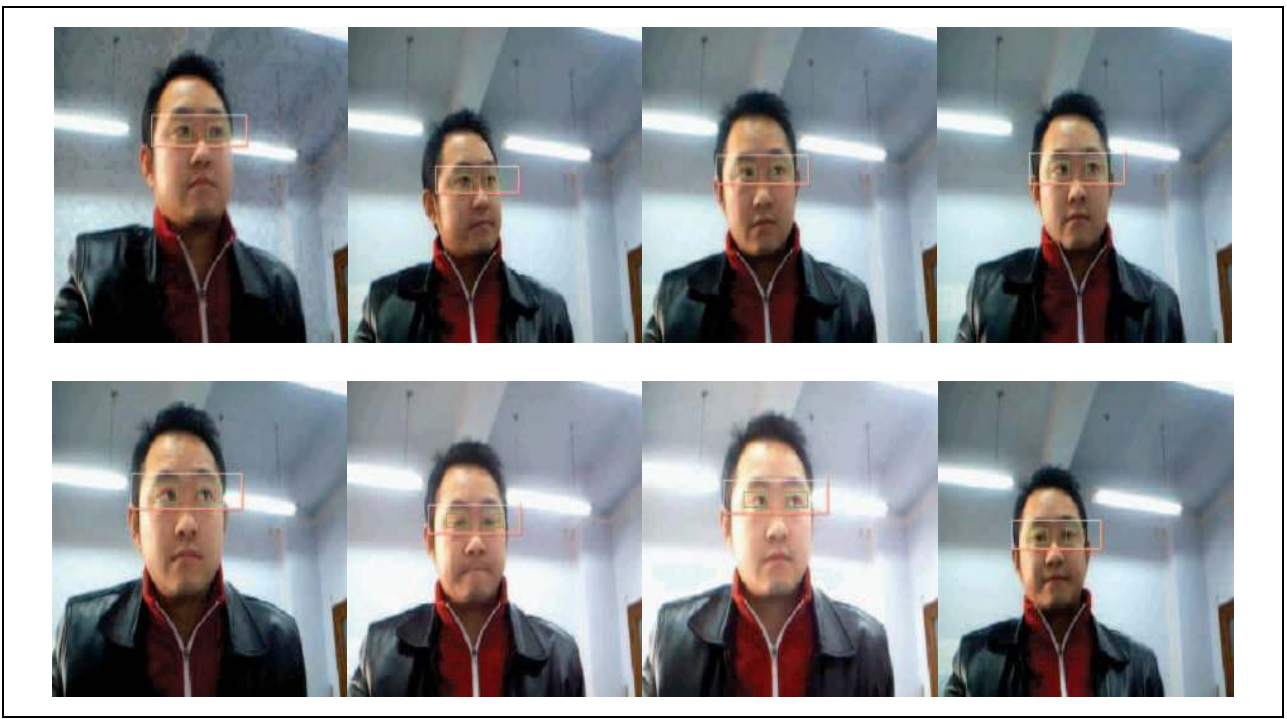




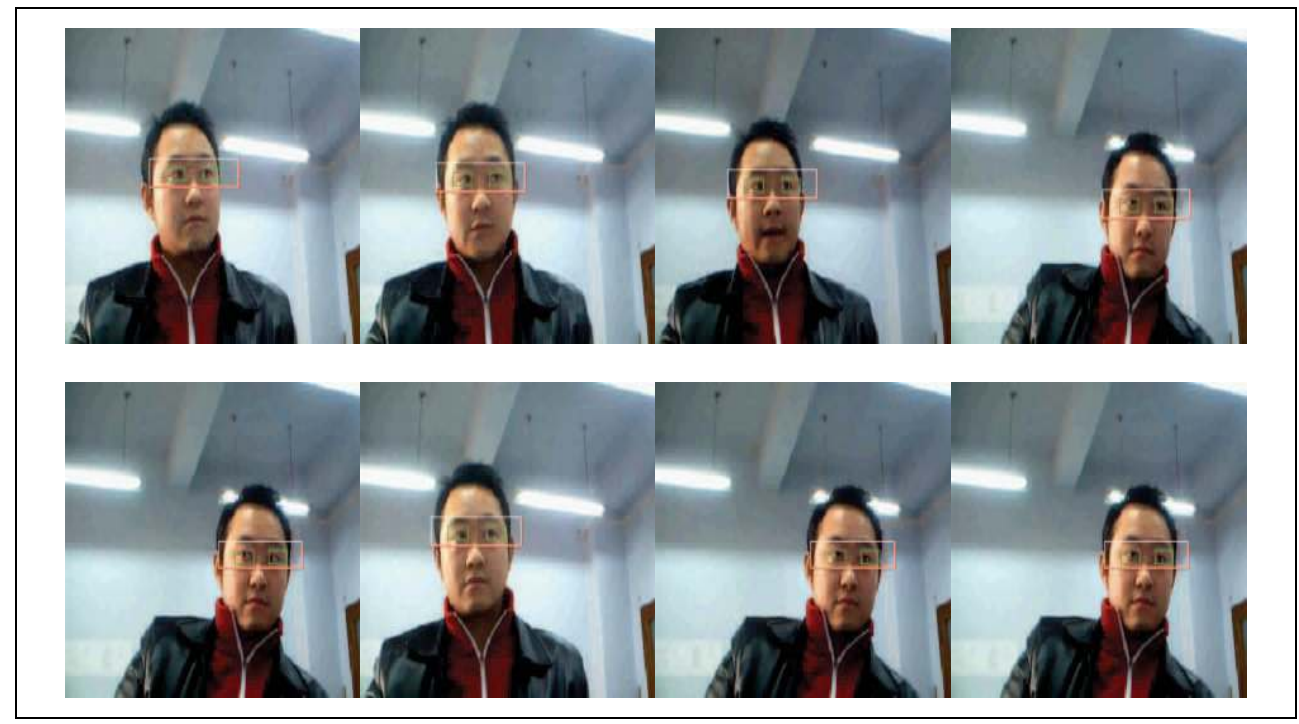

Fig. 1. Eye tracking using STFDEKF algorithm

\begin{tabular}{|l|c|c|c|}
\hline & Video 1 (without glasses) & Video2 (glasses) & Video3(long hair) \\
\hline Total Frames & 1999 & 2941 & 2889 \\
\hline Tracking Failure & 9 & 16 & 18 \\
\hline Correct Rate & $99.45 \%$ & $99.35 \%$ & $99.4 \%$ \\
\hline Average Correct Rate & \multicolumn{3}{|c}{$99.4 \%$} \\
\hline
\end{tabular}

Table 1. Result of eye tracking using STFDEKF algorithm Correct Rate of eye tracking is defined as in equation (25).

$$
\text { Correct } \text { Rate }=\frac{\text { Total Frames }- \text { Tracking Failure }}{\text { Total Frames }}
$$

The following experimental results evaluate the performance of our proposed method and other eye tracking methods.

\begin{tabular}{|l|c|c|}
\hline Algorithm & Correct rate & remark \\
\hline Templates Match & $99.1 \%$ & Refer to (Horng et al., 2004) \\
\hline Kalman and mean shift algorithm & $99.1 \%$ & Refer to (Zhu et al., 2002) \\
\hline EKF tracking algorithm & $99 \%$ & \\
\hline STFDEK algorithm in this paper & $99.4 \%$ & Refer to Table.1 \\
\hline
\end{tabular}

Table 2. Comparison of eye tracking algorithms 
In order to qualitatively gauge performance and discuss resulting issues, we consider using the traditional measures of performance: the RMSE (Root Mean Square Error) and MSE (Mean Square Error). The simulation results of RMSE and MSE are depicted in Table 3.

\begin{tabular}{|l|c|c|}
\hline Algorithm & RMSE & MSE \\
\hline Kalman Filter algorithm & 0.13155 & 0.164661 \\
\hline EKF tracking algorithm & 0.1222 & 0.0904 \\
\hline STFDEK algorithm in this paper & 0.0989 & 0.0780 \\
\hline
\end{tabular}

Table 3. RMSE and MSE of eye tracking filtering algorithms

The results of above experiments indicate that the proposed method has better performance. So we can use STFDEK algorithm for eyes tracking.

\section{Conclusion}

This paper proposes a new eye tracking method using strong finite-difference Kalman filter. Firstly, strong tracking factor is introduced to modify priori covariance matrix to improve the accuracy of the eye tracking algorithm. Secondly, the finite-difference method is proposed to replace partial derivatives of nonlinear functions to eye tracking. From above deduction, the new strong finite-difference Kalman filter becomes very simple because of replacing partial derivatives calculation using finite-difference value. The last experiment results show that STFDEKF has more accuracy and covariance estimation, improves the robustness of target tracking, and can be used for the high nonlinear stochastic systems such as eye tracking.

\section{References}

Qiang, J.; Zhu, Z.W. \& Peilin Lan.(2004). Real-Time Nonintrusive Monitoring and Prediction of Driver Fatigue, IEEE Transactions on Vehicular Technology, VOL. 53, NO. 4, July 2004, pp.1052-1068, ISSN:0018-9545

Horng, W.B.; Chen, C.Y. \& Chang, Y.(2004). Driver Fatigue Detection Based On Eye Tracking and Dynamic Template Matching, Proceeding of the 2004 IEEE International Conference on Networking, Sensing \& Control, pp.7-12,ISBN:0-7803-8193-9, Taipei, Taiwan, March 21-23,2004

Dong, W.H. \& Wu,X.J.(2004). Driver Fatigue Detection Based on the Distance of Eyelid, Proceeding of the 2005 IEEE International Workshop VLSI Design \& Video Tech, pp.365368,ISBN:0-7803-9005-9, Suzhou, China, May 28-30,2005

P.Majaranta \& K.Raiha. (2002). Twenty year of eye typing: system and design issues, Proceeding in ACM eye tracking research and applications symposium, pp.15-22, ISBN: 158113-467-3, New Orleans, Louisiana,USA, March 2002

Laura A.; Granka \& Thorsten Joachims. (2004). Eye-Tracking Analysis of User Behavior in WWW Search, Proceeding of the 200 SIGIR, pp.478-479, ISBN:1-58113-881-4, Sheffield, South Yorkshire, UK, July 25-29, 2004 
Noton \& Stark, L.(1971). Scanpaths in accadic eye movements while viewing and recognizing patterns vision research, PMID: 5538847, Jan 22,1971

Takehiko Ohno; Naoki Mukawa \& Shinjiro Kawato.(2003).Just Blink Your Eyes: A HeadFree Gaze Tracking System, Proceeding of the 2003 CHI, pp.115-122, ISBN:1-58113825-3, Ft. Lauderdale, Florida, USA, April 5-10, 2003

John D. McCarthy; Jens Riegelsberger \& M. Angela Sasse.(2005). Commercial uses of eye tracking, Proceeding of the 2005 British HCI Group Annual Conference, Napier University, Edinburgh, Monday 5th September 2005

Li, D.H.; David Winfield \&Derrick J.(2005). A hybrid algorithm for video-based eye tracking combining feature-based and model-based approaches, Proceeding of the IEEE Vision for Human-Computer Interaction, 2005

Zhu, J. \& Yang, J.(2002). Subpixel eye gaze tracking, Proceeding of the 2002 IEEE conference on Automatic Face and Gesture Recognition, pp.124-129, ISBN:0-7695-1602-5, May 2002

Tohno; N.Mukawa \& A.Yoshikawa.(2002). Freegaze: a gaze tracking system for everyday gaze interaction, Proceeding of the 2002 Eye tracking research and applications symposium, pp.15-22, ISBN:1-58113-467-3, New Orleans, Louisiana, March 2002

Zhu, Z.W.; Qiang, J. \& Kikuo Fujimura.(2002). Combining Kalman Filtering and Mean Shift for Real Time Eye Tracking under Active IR Illumination, Proceeding of the 2002 International Conference on Pattern Recognition (ICPR'02), ISBN:0-7695-1695-X, 2002

Y. Ebisawa.(1995). Unconstrained pupil detecting technique using two light sources and the image difference method, Visualization and Intelligent Design in engineering and architecture,pages 79-89, 1995

C.H. Morimoto \& M. Flickner.(2000). Real-time multiple face detection using active illumination, Proceeding of the 4th IEEE International Conference on Automatic Face and Gesture Recognition, pp.8-13, ISBN:0-7695-0580-5, Grenoble, France, March 2000

Daugman.J.(1993). High confidence visual recognition of persons by a test of statistical independence, IEEE Transactions on Pattern Analysis and Machine Intelligence, vol. 15, no. 11, pp. 1148-1161, ISSN: 0162-8828

Nishino.K \& S. Nayar.(2004). Eyes for relighting, ACM SIGGRAPH 2004, vol. 23, no. 3, pp. 704-711, 2004

Michael Chau \& Margrit Betke.(2005). Real Time Eye Tracking and Blink Detection with USB Cameras, Boston University Computer Science Technical Report No. 2005-12

Zhou, D. H.; Xi, Y. G. \& Zhang, Z. J.(1991). A suboptimal multiple fading extended Kalman filter, Chinese J. Automation, pp.689-695, ISSN: CN11-2109/TP, 1991

Zhou, D. H. (1990). Fault detection and diagnostics for a class of nonlinear systems, Ph.D. dissertation, Shanghai Jiao Tong Univ., P.R. China, 1990.

Zhou, D. H.; Sun, Y.X. \& Zhang, Z. J.(1993). Extension of Friedland's Separate-Bias Estimation to Randomly Time-Varying Bias of Nonlinear Systems, IEEE TRANSACTIONS ON AUTOMATIC CONTROL, VOL. 38, NO. 8, AUGUST 1993 
Fan,W.B.; LIU, C.F. \& Zhang, S.Z.(2006). Improved Method of STRONG Tracking Extended Kalman Filter, Control and Decision Jan,2006

Zhou, D. H. \& Q.L.(2006). Strong Tracking Filter of Nonlinear Systems with Colored Noise, Journal of Beijing Institute of Technology, vol.17, no.3, pp.321-326,1997 


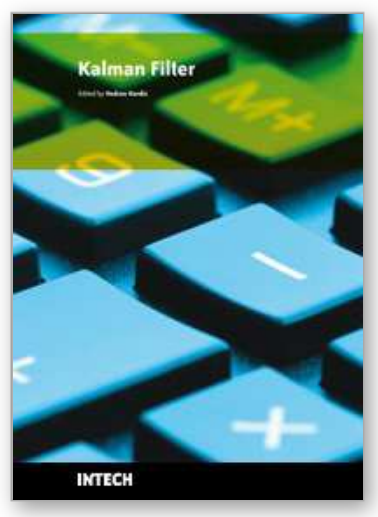

\author{
Kalman Filter \\ Edited by Vedran Kordic
}

ISBN 978-953-307-094-0

Hard cover, 390 pages

Publisher InTech

Published online 01, May, 2010

Published in print edition May, 2010

The Kalman filter has been successfully employed in diverse areas of study over the last 50 years and the chapters in this book review its recent applications. The editors hope the selected works will be useful to readers, contributing to future developments and improvements of this filtering technique. The aim of this book is to provide an overview of recent developments in Kalman filter theory and their applications in engineering and science. The book is divided into 20 chapters corresponding to recent advances in the filed.

\title{
How to reference
}

In order to correctly reference this scholarly work, feel free to copy and paste the following:

Zutao Zhang and Jiashu Zhang (2010). Application of a Strong Tracking Finite-Difference Extended Kalman Filter to Eye Tracking, Kalman Filter, Vedran Kordic (Ed.), ISBN: 978-953-307-094-0, InTech, Available from: http://www.intechopen.com/books/kalman-filter/application-of-a-strong-tracking-finite-difference-extendedkalman-filter-to-eye-tracking

\section{INTECH}

open science | open minds

\author{
InTech Europe \\ University Campus STeP Ri \\ Slavka Krautzeka 83/A \\ 51000 Rijeka, Croatia \\ Phone: +385 (51) 770447 \\ Fax: +385 (51) 686166 \\ www.intechopen.com
}

\author{
InTech China \\ Unit 405, Office Block, Hotel Equatorial Shanghai \\ No.65, Yan An Road (West), Shanghai, 200040, China \\ 中国上海市延安西路65号上海国际贵都大饭店办公楼 405 单元 \\ Phone: +86-21-62489820 \\ Fax: +86-21-62489821
}


(C) 2010 The Author(s). Licensee IntechOpen. This chapter is distributed under the terms of the Creative Commons Attribution-NonCommercialShareAlike-3.0 License, which permits use, distribution and reproduction for non-commercial purposes, provided the original is properly cited and derivative works building on this content are distributed under the same license. 